\title{
CORRESPONDENCE
}

Abdominal aortic aneurysms

Jackie Walton, BSC, and J Collin, FRCS ......... 1414

Laboratory control of oral anticoagulants

R V Majer, MRCP, and others ................. 1414

Intensive care: a specialty or a branch of anaesthetics?

JF Searle, FFARCS, and Sheila Willatts, FFARCS 1415

Angie's overdose

SJEllis, MB, and Susan Walsh, MB ........... 1415

Sue's breast lump

RB Balland, FRCS, and H B Ross, FRCS .

$\beta$ Endorphin: a factor in "fun run" collapse?

DMW deCVeale, MB ................... 1415

$\mathrm{DM} \mathrm{W}$ de CVeale, $\mathrm{MB}$....................................
Corticosteroids and bone mass in asthma

D M Reid, MRCP, and others ................ 1416

A forgotten factor in pelvic inflammatory

disease: infection in the male partner

$\mathrm{CMStacey,} M B$, and others .
Social class, non-employment, and chronic

illness

M J Scott, MB; Sara Arber, MSC.

Screening for congenital dislocation of the hip

NM PClarke, FRCS........................... 1417

Screening for atlantoaxial instability

GHowells, MRCGP ........................... 1417

RAWPing general practice

IGCrossman, FDS .

Effect of combined implants of oestradiol and testosterone on libido in postmenopausal women

L Appleby, MRCPSYCH, and Julia Montgomery, MRCs; Jean Hailes, MB, and others....

General managers and consultants

RHopkins, MRCS.
Auditory rehabilitation-should we listen to the patient?

HHLangston, FRCS ........................ 1418

FRCS (UK)?

MJones, FRCS

Points Clinical trials and the acceptance of uncertainty (Sir Austin Bradford Hill);

Effects of breast conservation on psychological morbidity (D J Fairlamb; H Ellis); Why the excess mortality from psychiatric illness? (C J Bruton); Syringe driver in terminal care $(R$ J Dickson and PS B Russell); Diabetogenic effect of cyclosporin (I Hulinský); Terms and conditions of service (J B Wood); Measles and the government (D Walker and H Carter); Dangers from dissolution of latex in Celestin endooesophageal tube(CEFanghanel) .............

All letters must be typed with double spacing and signed by all authors.

- No letter should be more than 400 words.

- For letters on scientific subjects we normally reserve our correspondence columns for those relating to issues discussed recently (within six weeks) in the BMF.

- We do not routinely acknowledge letters. Please send a stamped addressed envelope if you would like an acknowledgment.

- Because we receive many more letters than we can publish we may shorten those we do print, particularly when we receive several on the same subject.

\section{Abdominal aortic aneurysms}

SIR,-Mr Gowland Hopkins (28 March, p 790) and Dr Janet Powell and Professor R M Greenhalgh ( 2 May, p 1161) are concerned by the cost of ultrasound screening for abdominal aortic aneurysm in all elderly men and propose alternative forms of selective screening.

Mr Gowland Hopkins suggests palpating the abdomen of men over 50 with ultrasonography for doubtful cases. Our screening programme for unselected men aged 65-79 comprises both abdominal palpation and ultrasound examination. Abdominal palpation has so far failed to detect any of the $2 \cdot 8 \%$ of aneurysms yielded by ultrasound examination and has produced $7 \cdot 1 \%$ false positive findings. Robicsek has shown that even in patients with a suspected aneurysm abdominal palpation confirms the diagnosis in less than one third, while in patients . with strongly suspicious palpatory findings no aneurysm is present in more than a half.' ${ }^{\prime}$

Dr Powell and Professor Greenhalgh suggest screening the smoking relatives of female and younger patients with aneurysms. There have been only eight women among the 93 patients surviving abdominal aortic aneurysm surgery in Oxford in the past 18 months and only $5 \cdot 4 \%$ of patients were aged under 60. Detailed family histories from our last 28 patients yielded 119 first degree relatives, of whom 84 were already permanently beyond the reach of surgery. Of the 35 surviving siblings our patients would volunteer no addresses for 24, either because all contact had been lost or because they were too old, too frail, or too far away for us to help them. We have obtained the addresses of 11 people, five of whom live in the Oxford region. If we achieve the usual response rate to invitations for examination we can expect to screen one first degree relative for every 15 patients we operate on for an aortic aneurysm.

The screening methods suggested by $\mathrm{Mr}$ Gowland Hopkins and Dr Powell would both undoubtedly be cheap but also ineffectual.

Nuffield Department of Surgery,

JACKIE WALTON John Radcliffe Hospital, Oxford OX39DU

1 Robicsek F. The diagnosis of abdominal aneurysms. Surgery 1981;8:275-6.

\section{Laboratory control of oral anticoagulants}

SIR,-Dr L Poller (9 May, p 1184) is rightly concerned that after the withdrawal of the Manchester human brain thromboplastin anticoagulant control in this country may suffer. We would disagree, however, that it is necessary to use a reagent with an international sensitivity index of 1.0-1.2.

For the past year we have used Diagnostic Reagents rabbit brain thromboplastin, which has an index of $1 \cdot 4$. Using a large number of patient samples and the Diagnostic Reagents thromboplastin as the reference preparation we have published our results, comparing this reagent with the Manchester human brain thromboplastin and the Manchester rabbit brain thromboplastin. The results gave international sensitivity index values very close to the manufacturers' stated values. ${ }^{\prime}$

Stimulated by Dr Poller's leading article, we looked at other aspects of our practice. We check our control times daily on fresh plasma pooled from four to eight normal donors. The weekly mean of control times for one year before we changed reagent, using Manchester human brain thromboplastin, was 11.7 (SD 0.352) seconds. The weekly mean of control times using Diagnostic Reagents thromboplastin for a year was 13.6 $(0 \cdot 274)$ seconds. During the year we have used 12 batches of Diagnostic Reagents thromboplastin and have seen neither a greater variation in control times than when using Manchester thromboplastin nor a change in control times between batches of Diagnostic Reagents thromboplastin.

From batches of frozen control plasma, one from 20 normal donors and another from 20 well controlled patients receiving anticoagulants, the international normalised ratio is calculated daily. The mean value for one entire batch of frozen control samples (two months) using Manchester human brain thromboplastin was $2 \cdot 3(0 \cdot 114)(39$ readings). The mean value of a subsequent entire batch of frozen samples using Diagnostic Reagents thromboplastin over two months was $2 \cdot 0(0 \cdot 119)$. The international normalised ratios are not comparable because they relate to different batches of controls, but the standard deviations are very close.

From our anticoagulant clinic 13 stable patients receiving long term treatment with warfarin, who had not required any change in dose for a year before and a year after the change in reagent, were identified. With Manchester thromboplastin the range of eight results for the international normalised ratio was 2.2 to 2.8 (mean 2.5 ), with a range of standard deviation of 0.196 to 0.454 (mean $0: 299$ ). With Diagnostic Reagents thromboplastin the range of results for the ratio was 2.3 to 3.0 (mean $2 \cdot 6$ ), with standard deviations ranging from $0 \cdot 192$ to 0.444 (mean 0.34 ). Again the values are very close. No change in anticoagulant dose was 
required because of a change in thromboplastin.

We are satisfied that the change from human to rabbit brain thromboplastin has not been deleterious and that the reagent we are using is well calibrated and of high quality.

R V MAJER P J GREEN

P WEIR

\author{
Central Laboratory, \\ St Mary's Hospital, \\ Portsmouth PO3 6AG \\ 1 Weir P, Green PJ. Rabbit (to replace human) brain material in \\ anticoagulant control. Lancet 1986;ii:1335.
}

\section{Intensive care: a specialty or a branch of anaesthetics?}

SIR,-It is remarkable that over two months elapsed before there was a published comment on Professor Hugh Dudley's leading article on intensive care training (21 February, p 459). We have been reticent in replying because our own proposals for a pilot senior registrar training scheme in the South Western region were being considered by the interfaculty/collegiate liaison group on intensive therapy. We now understand that our scheme has been approved, and we therefore welcome Professor Iain Ledingham's letter (25 April, p 1095), with its emphasis on the importance of encouraging without delay training programmes recommended by the liaison group.

There are two main obstacles to achieving this. The first is funding. The liaison group was set up jointly by the Faculty of Anaesthetists, the Royal Colleges of Surgeons of England and Scotland, and the Royal College of Physicians. Its proposals have been accepted by the conference of royal colleges and their faculties. The agreement achieved is remarkable if not unique. It is difficult to believe that the conference cannot persuade the Department of Health and Social Security of the need to fund a small number of posts, unless those who have supported the liaison group publicly are privately hoping that lack of funds will ensure its premature death.

The second problem is the anxiety among some anaesthetists that the proposals will undermine their role in intensive care and interfere with the training of anaesthetic registrars and senior registrars. For over 20 years anaesthetists have borne the main burden of intensive care in this country, and they will continue to do so. Trainees in other disciplines, however, also wish to pursue a career in intensive care. Is it essential that they must have completed all three parts of the FFARCS examination before being allowed to do so? We think not. The liaison group's proposals and our own South Western training scheme are designed to improve the training of those doctors who wish to have intensive care as a major component of their consultant career, and who include anaesthetists. Furthermore, the presence of a senior registrar in an intensive care unit may actually improve the training of registrars as he or she will be more readily available to teach than many consultants.

So far as senior registrars in anaesthesia are concerned, it should be possible to maintain the amount of training in intensive care, even though there may already be a senior registrar on the intensive care unit in a hospital to which the new trainee rotates. In many regions, however, training of senior registrars in intensive care is limited by the demand for training in subspecialties within anaesthesia. It is essential that existing anaesthetic senior registrars continue to receive such training as many will undertake on call intensive care work as consultants.

Many anaesthetists support these proposals.
Some who oppose them misunderstand them. A few are unwilling formally to open intensive care training to disciplines other than anaesthesia. The proposals arose out of the need to improve the care of critically ill patients in Britain. It saddens us that parochial concerns may defeat this laudable objective.

Royal Devon and Exeter Hospital,

JOHN F SEARLE Exeter EX2 5DW

Sheila Willatts

Bristol Royal Infirmary,

Bristol BS2 8HW

\section{Angie's overdose}

SIR,-Dr Stephen Platt's study (11 April, p 954) failed to show a strong imitation effect after televised parasuicide. His data were obtained by letter from accident and emergency departments throughout Britain. Only $41 \%$ of the original total provided usable returns for 1985 and 1986 Collecting numbers of overdoses from accident and emergency registers is tedious and time consuming, and there must be doubt about the accuracy of responses obtained from poorly motivated administrative staff. For the Hackney Hospital Platt recorded 11 overdoses during the experimental period, while a more careful look at the records reveals 22 .

Dr Platt's results do show a significant increase in the number of overdoses for the 18 days after the first screening of Angie's overdose compared with the 18 days before it $(p<0.001$, sign test). The question remains whether there is a causal relation between the EastEnders programme and this finding.

By choosing the week before the overdose as his control week Platt may be underestimating the initiative effect by including subjects who had been influenced by Angie's plight but who beat her to the inevitable conclusion. Although the overdose was shown on Thursday 27 February 1986, the plot had been building up to it for some time. Angie's overdose was not so much an isolated event but the culmination of a process-as overdose is for many people.

Although the medical and scientific community should understand the significance of a single negative study, the Independent Broadcasting Authority and the British Broadcasting Corporation may misinterpret these results as a licence to continue the gratuitous exploitation of parasuicide in soap opera. Since our publication of the initial observed increase in overdoses following Angie's ${ }^{1}$ the IBA may have thought twice about the portrayal of self harm, but not so the BBC. On 17 October 1986 Amanda in Dynasty took an overdose. She was showered with affection and given a diamond necklace for her pains. The only other way to obtain a diamond necklace in Hackney is to rob a jeweller.

Simon J Ellis

Frimley Park Hospital,

SUSAN WALSH

Frimley Park Hospi
Surrey GU16 5UJ

1 Ellis SJ, Walsh S. Soap may seriously damage your health. Lancet 1986;i:686.

\section{Sue's breast lump}

SIR,-Dr Stephen Platt assessed the influence of an attempted suicide in EastEnders on the number of subsequent overdoses (11 April, p 954).

Another character in the same programme has recently had a breast lump diagnosed and treated.

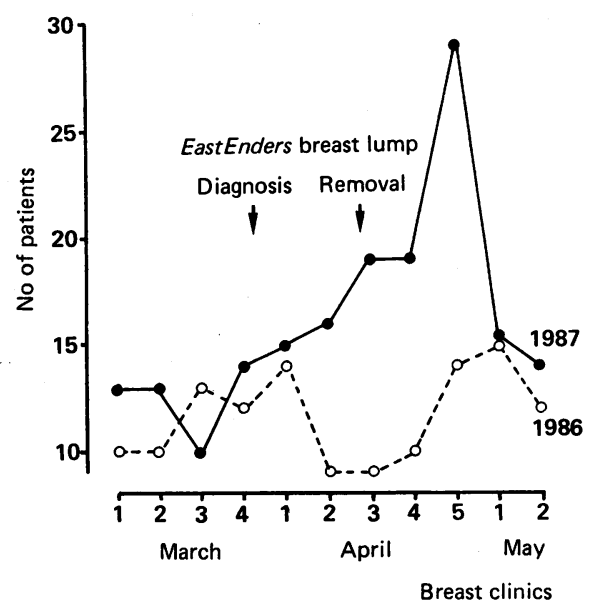

Number of patients attending successive weekly breast clinics.

The impact of this on new referrals to a weekly breast clinic is shown in the figure (most new patients are seen within a week of visiting their general practitioner). Forty of the patients who attended the fourth or fifth clinic in April were questioned about EastEnders. Twenty six had either watched the programme or knew that someone in the programme had recently had a breast lump. Six patients claimed that they had attended directly as a result of the programme. Five were ultimately diagnosed as having malignant lesions, 27 as having benign lesions, and eight as having no abnormality. One of the patients with a carcinoma said that she would not have sought advice had it not been for EastEnders.

Although the "imitation phenomenon" may not have been proved with regard to Angie's suicide attempt, Sue Osmond's breast lump appears to have influenced the number of patients presenting to a specialised breast clinic. The effect on clinical workload of medical problems shown in a television programme which is regularly watched by over 20 million people should not be discounted. By increasing awareness programmes such as EastEnders might lead to earlier attendance at screening or treatment clinics.

R B GALLAND H B Ross

Royal Berkshire Hospital,

Reading RG1 5AN

\section{$\beta$ Endorphin: a factor in "fun run" collapse?}

SIR,-Dr G Dale and colleagues (18 April, p 1004) claim that a high concentration of circulating $\beta$ endorphin is associated with the collapse of some men during "fun runs."

Runners who collapse are usually those who are ill prepared and undertrained, and an increased plasma concentration of $\beta$ endorphin is a nonspecific response to acute stress. ${ }^{1}$ Plasma $\beta$ endorphin and adrenocorticotrophic hormone are derived from a common precursor in the pituitary, pro-opiomelanocortin, which secretes both hormones in response to the same stress. ${ }^{2}$ Thus cortisol concentrations are also likely to be increased in runners who collapse. The exact role of plasma $\beta$ endorphin in man is not known, but it may modulate the release of circulating catecholamines and the renin-aldosterone system. ${ }^{3}$ As plasma $\beta$ endorphin is fairly impermeable to the blood-brain barrier except at the hypothalamus it cannot be implicated in the "runner's high" or in driving a runner to collapse. ${ }^{4}$

Central changes in opioid peptide concentra- 